\title{
Brucellosis: A Case Report and Literature Review
}

\author{
Mahnaz Hakeem ${ }^{1}$, Sana Saeed ${ }^{2}$
}

\begin{abstract}
Brucellosis is a major cause of febrile illnesses in areas with high exposure to animals or unpasteurized milk and dairy products. It may manifest with multisystem clinical presentations. Our patient was a six-year-old boy who was presented in emergency room with a prolonged history ( 3 weeks) of high-grade fever with chills, not responding to antibiotics. Upon inquiry, the history revealed the use of unpasteurized goat milk and contact with goats at home. Upon investigation, serum antibodies for brucellosis were raised (1:640). He was started on combination therapy with rifampicin and doxycycline. He became afebrile within two days so was discharged home. We found that an ineffective history could lead to delay in diagnosis and management. Clinicians should have high index of suspicion for brucellosis in children.

Keywords: Brucellosis, Pyrexia of unknown origin, Zoonotic disease.

Journal of Postgraduate Medicine Education and Research (2019): 10.5005/jp-journals-10028-1328
\end{abstract}

\section{BACKGROUND}

Brucellosis also known as Mediterranean fever, Malta fever, or Undulant fever is a zoonotic disease caused by bacteria genus Brucella. ${ }^{1}$ Population at risk includes farmers, animal handlers, and laboratory workers. Brucellosis may remain asymptomatic or manifest as chronic febrile illness. Failure to obtain an adequate history, lack of a thorough examination, delay in obtaining appropriate investigations, and difficulties in interpretation of results often result in missed diagnoses. ${ }^{2}$ Prevention is ensured by massive livestock vaccination and improving hygiene practices and pasteurization of milk products. We are reporting a case of a child who was diagnosed as having brucellosis after 3 weeks of initial presentation due to paucity in obtaining clinical history. Moreover, we have also reviewed the studies on clinical presentation and treatment of brucellosis.

\section{Case Description}

A 6-year-old boy was brought into the emergency department (ED) with a complaint of fever for three weeks. The fever was reported high grade (axillary temperature of $39-40^{\circ} \mathrm{C}$ ) with chills and rigors, occurring 3-4 times in day. There were no other associated symptoms. He was being managed in multiple hospitals with intravenous ceftriaxone, amikacin, and artemether but the fever persisted. Past history and family history were found to be insignificant.

Medical examination in ED revealed temperature of $40^{\circ} \mathrm{C}$ with a heart rate of 130 beats/min. There were no skin rash, lymphadenopathy, or hepatosplenomegaly. Laboratory results showed leucopenia with normal neutrophil and lymphocyte counts $\left(4.5 \times 10 \times 10^{9} / \mathrm{L}\right.$, neutrophil $45.6 \times 10 \times 10^{9} / \mathrm{L}$, lymphocyte $\left.47.6 \times 10 \times 10^{9} / \mathrm{L}\right)$. Blood and urine cultures showed no infection. Malarial parasite and ICT-malaria were negative. He was admitted for the documentation and management of fever on intravenous ceftriaxone and oral paracetamol. On probing the history, it was found that the child had been drinking unboiled goat milk since a couple of years and has direct contact with these goats at home. Bearing in mind the possibility of brucellosis, serum antibodies were sent. The antibody titer was reported 4 times higher (1:640) than the normal $(<1: 80)$. He was started on combination therapy of rifampicin $10 \mathrm{mg} / \mathrm{kg} /$ day and doxycycline $4 \mathrm{mg} / \mathrm{kg} /$ day. $\overline{1,2 \text { Department of Paediatrics and Child Health, Aga Khan University }}$ Hospital, Karachi, Pakistan

Corresponding Author: Sana Saeed, Department of Paediatrics and Child Health, Aga Khan University Hospital, Karachi, Pakistan, Phone: +92 213 4862265, e-mail: sana.saeed@aku.edu

How to cite this article: Hakeem M, Saeed S. Brucellosis: A Case Report and Literature Review. J Postgrad Med Edu Res 2019;53(3):126-127.

Source of support: Nil

Conflict of interest: None

\section{Outcome and Follow-up}

Within 48 hours of starting treatment, he became afebrile so he was discharged home with the plan to continue the treatment for a total of 6 weeks. Upon follow-up, after 2 weeks, there were no documented episodes of fever.

\section{Discussion}

There are many causes of unexplained fever in children. Etiologies include rare diseases but also include common illnesses presenting unusually. Brucellosis is a zoonotic disease, commonly encountered in areas with high exposure to animals and use of unpasteurized milk. Other possible mechanisms to acquire brucellosis include vertical transmission and occupational exposure in microbiology laboratories. Studies have shown prevalence of brucellosis in children as high as $20-25 \%$. ${ }^{3}$ Since the primary mode of transmission in Pakistan remains animal exposure and unpasteurized milk consumption, there are no local data regarding disease prevalence in the pediatric population. However, seroprevalence of high-risk occupational group in Pakistan was found to be $21.7 \%{ }^{4}$

It is often considered as a diagnostic dilemma in both endemic and nonendemic areas due to its vague presentations, including fever, diaphoresis, malaise, headache, anorexia, and body aches (Table 1). Fever is characterized as regular remissions giving its characteristic name "undulant fever." Fever and arthralgia are reported to be the most common symptom and sign in brucellosis. ${ }^{5}$ Physical examination could be marked by arthritis, lymphadenopathy, and hepatosplenomegaly. Multisystem involvement is also reported including joints, bones, 
Brucellosis

Table 1: Clinical presentation and outcome of brucellosis in children

\begin{tabular}{|c|c|c|c|c|c|c|}
\hline Authors & $\begin{array}{l}\text { Age/ } \\
\text { gender }\end{array}$ & Risk factor & Presentation & $\begin{array}{l}\text { Antibody } \\
\text { titer }\end{array}$ & Treatment & Outcome \\
\hline Farhan et al. ${ }^{12}$ & 13 years/F & Horse riding & CNS & $1: 320$ & $\begin{array}{l}\text { Doxycycline, rifampicin } \\
\text { and streptomycin }\end{array}$ & Cured \\
\hline Aghdam et al. ${ }^{13}$ & 8 years/M & Unpasteurized dairy & Recurrent epistaxis & $1: 320$ & TMP-SMX, rifampicin & Cured \\
\hline Yaman et al. ${ }^{14}$ & $\begin{array}{l}\text { I. } 4 \text { years/M } \\
\text { II. } 16 \text { years/M } \\
\text { III. } 8 \text { years/M }\end{array}$ & Unpasteurized dairy & Secondary HLH & $1: 320$ & $\begin{array}{l}\text { TMP-SMX, rifampicin } \\
\text { IVIG }\end{array}$ & All cured \\
\hline Metin et al. ${ }^{15}$ & 11 years/F & Unpasteurized dairy & Hemorrhagic fever & $1: 320$ & Doxycycline, rifampicin & Cured \\
\hline Akbayram et al. ${ }^{16}$ & 7 years/F & Unpasteurized dairy & $\begin{array}{l}\text { Thrombotic thrombo- } \\
\text { cytopenic purpura }\end{array}$ & $1: 1280$ & $\begin{array}{l}\text { TMP-SMX, rifampicin } \\
\text { Methylprednisolone }\end{array}$ & Expired \\
\hline
\end{tabular}

CNS, central nervous system; HLH, hemophagocytic lymphohistiocystosis; IVIG, intravenous immunoglobulin TMP-SMX, trimethoprim-sulfamethoxazole

gastrointestinal, and central nervous system (CNS). Neurobrucellosis is found in $0.8 \%$ children. ${ }^{6}$

The diagnosis of brucellosis is usually based on serology, including blood culture and antibody titers. Sensitivity of blood culture varies from 53 to $90 \%$. In case of negative blood cultures, one may opt for bone marrow cultures, which is gold standard, having sensitivity as high as $97 \% .^{7}$ Serum agglutination test is considered as an alternative for the diagnosis of brucellosis traditionally. A titer of 1:>160 is favorable for the diagnosis of brucellosis. Neuroimaging reveals inflammatory changes and raised intracranial pressure with ventricular dilatation or rarely as a space-occupying lesion. ${ }^{8}$ Hematological findings in brucellosis are reported to be either normal or include anemia, leukopenia, neutropenia, thrombocytopenia, and pancytopenia. ${ }^{9}$

Treatment of brucellosis is preferred to be started in combination therapy in order to avoid relapse. Doxycycline is used in combination with rifampicin or gentamicin in uncomplicated brucellosis cases of greater than 8 years of age or rifampicin and septran or gentamicin for age less than 8 years. Trimethoprim-sulfamethoxazole replaces tetracycline in children $<8$ years of age. Duration of therapy is usually 4-6 weeks. Addition of gentamicin for initial 7-10 days along with doxycycline and rifampicin is associated with lower relapse rates. ${ }^{10}$ Neurobrucellosis is treated with the combination of an aminoglycoside, rifampin, and doxycycline, TMP-SMX.

Prognosis in brucellosis is good in majority of patients, if treated properly but it has been reported with severe neurodevelopmental sequelae in cases with neurobrucellosis. ${ }^{11}$ Unfavorable outcome is usually observed in spondylitis and neurobrucellosis.

\section{ConcLusion}

Brucellosis resembles a number of diseases in its multisystem involvement. Clinicians should have a high index of suspicion when encountering febrile patients with known consumption of unpasteurized dairy products as rapid diagnosis, and the treatment of brucellosis is the key to decrease the morbidity.

\section{References}

1. GUL ST, Khan A. Epidemiology and epizootology of brucellosis: a review. Pakistan Vet J 2007;27(3):145-151.

2. Zhen Q, Lu Y, et al. Asymptomatic brucellosis infection in humans: implications for diagnosis and prevention. Clin Microbiol Infect 2013;19(9):E395-E397.

3. Mantur BG, Akki AS, et al. Childhood brucellosis-a microbiological, epidemiological and clinical study. J Trop Pediatr 2004;50(3): 153-157.

4. MukhtarF. Brucellosis in a high risk occupational group:seroprevalence and analysis of risk factors. J Pak Med Assoc 2010;60(12):1031-1034.

5. Shaalan MA, Memish ZA et al. Brucellosis in children: clinical observations in 115 cases. Int J Infect Dis 2002;6(3):182-186.

6. Lubani MM, Dudin KI, et al. Neurobrucellosis in children. Pediatr Infect Dis J 1989;8(2):79-82.

7. Gotuzzo E, Carrillo C, et al. An evaluation of diagnostic methods for brucellosis-the value of bone marrow culture. J Infect Dis 1986;153(1):122-125.

8. Budnik I, Fuchs I, et al. Unusual presentations of pediatric neurobrucellosis. Am J Trop Med Hyg 2012;86(2):258-260.

9. Sachdev A, Vohra R, et al. Acute brucellosis of childhood: a case report with unusual features. Indian Pediatr 2001;38(12):1421-1425.

10. Mile B, Valerija K, et al. Doxycycline-rifampin versus doxycyclinerifampin-gentamicin in treatment of human brucellosis. Trop Doct 2012;42(1):13-17.

11. Bouza E, Garcia de la Torre M, et al. Brucellar meningitis. Rev Infect Dis 1987;9(4):810-822.

12. Farhan N, Khan EA, et al. Neurobrucellosis: A report of two cases. J Pak Med Assoc 2017 Nov;67(11):1762-1763.

13. Aghdam MK, Davari K, et al. Recurrent epistaxis and bleeding as the initial manifestation of brucellosis. Acta Medica Iranica 2016;54(3):218-219.

14. Yaman Y, Gözmen S, et al. Secondary hemophagocytic lymphohistiocytosis in children with brucellosis: report of three cases. J Infect Dev Ctries 2015 Oct 29;9(10):1172-1176

15. Metin $\mathrm{O}$, Teke TA, et al. A case of brucellosis mimicking CrimeanCongo hemorrhagic fever. J Infect Public Health 2015;8(3):302-304.

16. Akbayram S, Dogan $M$, et al. Thrombotic thrombocytopenic purpura in a case of brucellosis. Clin Appl Thromb Hemost 2011;17(3):245-247. 